\title{
Complications of Thyroid Surgery: How to Avoid Them, How to Manage Them, and Observations on Their Possible Effect on the Whole Patient
}

\author{
Tom Reeve, A.C., M.B., ${ }^{1}$ Norman W. Thompson, M.D. ${ }^{2}$ \\ ${ }^{1}$ Endocrine Surgical Unit, Department of Surgery, University of Sydney, Wallace Freeborn Block, Royal North Shore Hospital, St. Leonards, \\ New South Wales 2065, Australia \\ ${ }^{2}$ University of Michigan Medical Center, 1500 E. Medical Center Drive, Ann Arbor, Michigan 48109, USA
}

\begin{abstract}
Surgery of the thyroid takes place in an area of complicated anatomy and in which a number of vital physiologic functions and special senses are controlled. Thyroidectomy rarely is associated with mortality; but unless the surgeon performing it is well trained in operative surgery and is knowledgeable of the gland and its function, pathology, and anatomy, excellent results cannot be achieved. Failure to observe cardinal surgical principles may result in legal difficulties, which can be avoided. It is well to observe the principles and avoid problems. We address this issue herein.
\end{abstract}

Surgical procedures have the potential for complications, and thyroidectomy is no exception [1]. However, when thyroidectomy is performed under optimal conditions, within a milieu of sound anatomic and physiologic knowledge, combined with meticulous operative skill by surgeons who have been trained to perform the operation and who are comfortable performing it, the incidence of complication should be at a minimum; complications are more often dependent on physical factors, such as a large or intrathoracic goiter, extensive malignancy, and whether the operation is a primary or secondary procedure [2]. Social factors such as long waiting times to enter hospital and reduced operating room capacity may also affect the outcome, particularly if the delays result in a more advanced malignancy [2].

The operation requires removal of pathologic and normal thyroid tissue. The mindset involved in the operation is best modified to understand the proposition that if the thyroid is carefully devascularized the gland can be totally separated from its surrounding tissues and literally "falls out." This approach leads to the least damage to both the gland surface and the surrounding structures [3]. If these conditions are difficult to meet, it may be preferable, if possible, to refer the patient to a facility where optimal requirements for thyroid surgery can be met.

When complications occur they fall into several categories, including errors of omission or commission; and they may be inevitable or totally unanticipated. They may occur during the preoperative, operative, or postoperative phase; and they adversely affect anatomic structures, physiologic function, or both.

Correspondence to: T.S. Reeve, A.C., M.B.
The surgeon must be on the qui vive for such eventualities and "head them off at the pass."

\section{Preoperative Evaluation}

\section{History and Physical Examination}

A carefully obtained history that emphasizes symptoms, general physical status, genetic abnormalities, drug intake (particularly aspirin or other anticoagulants), response to previous surgery, and any previous abnormal bleeding problems are important factors to elicit and consider in a workup directed toward thyroidectomy. A thorough physical examination can alert the surgeon to possible preoperative and intraoperative difficulties and allow steps to be taken to reduce problems by making appropriate referral to an endocrinologist, cardiologist, radiologist, or anesthesiologist; appropriate steps can be taken before operation. As part of the first consultation it is important on professional grounds to ensure that the patient is fully informed about the disease and its management, which allows the patient to make informed decisions about the advice given by the surgeon. It can also help to inform patients about suitable literature that can give them a sound insight into their problem.

To ensure euthyroidism, thyroid function tests are essential, if thyroid storm is to be avoided in thyrotoxic patients [4]. The third-generation thyroid-stimulating hormone (TSH) determination has become the most sensitive single study. The appropriate use of antithyroid drugs or $\beta$-blockade preoperatively is mandatory in the thyrotoxic patient. If $\beta$-blockers have been used alone, their continuance for an appropriate period postoperatively is also mandatory [5]. The surgeon must also be aware of the patient who is hypothyroid, which is a possible indicator of malignancy or Hashimoto's disease; pretreatment with levothyroxine to the point of euthyroidism before operation is appropriate. Standard blood tests, including a serum calcium assay, should always be performed. If concomitant hyperparathyroidism is detected, which is not a rarity, a planned exploration of all four parathyroids is clearly indicated. Chest radiography or a mediastinal computed tomography (CT) scan should be done if any particular abnor- 
mality is suspected in regard to local anatomic distortion or substernal extension of the thyroid, or for metastatic disease if malignancy is diagnosed or suspected. Scintiscans are not done routinely and are reserved for patients with a suppressed TSH, which could be caused by Grave's disease, thyroiditis, or one or more hyperfunctioning ("hot") nodules. Ultrasonography (US) may be of occasional assistance, especially if accurate measurement is crucial to a particular study [6].

The cystic or solid nature of a lesion can usually be determined by fine-needle aspiration (FNA), without adding to the financial burden of the US examination. FNA should be used selectively in those with single nodules and multinodular goiter demonstrating a dominant nodule. A cytologic result is useful in assisting decision-making about the operative indication and choice of procedure. FNA has become the single most important diagnostic study in patients with thyroid nodules [7].

\section{Larynx}

Examination of the larynx by indirect laryngoscopy provides knowledge of the integrity of laryngeal function; it is best carried out by a third party, preferably an otolaryngologist. The same observer should repeat the examination postoperatively. Although it is frequently recommended, some surgeons question the need for it routinely in patients without previous operation and no change in voice quality [8]. Occasionally, a paralyzed cord is found preoperatively in a patient who has achieved full vocal cord compensation and has a normal voice. This finding alerts the surgeon to a special problem, and the medicolegal value of such knowledge is clear. Whether a surgeon checks for this infrequent concurrence in the asymptomatic patient, indirect laryngoscopy should always be done in reoperative cases, regardless of voice quality, because compensated vocal cord paralysis is not a rarity in this situation. It is useful to have the anesthesiologist check the vocal cords when the endotracheal tube is removed. The normal movement of the cords at that time is predictive of normal ongoing function, even if the vocal cord develops delayed paresis. Repeat indirect laryngoscopy allows accurate review of cord function, rather than presuming normal function if the voice appears normal.

In summary, careful planning is undertaken to ensure smooth operative progress and to allow the surgeon to be alerted to any out of the ordinary findings. It is wise to make the anesthesiologist aware of any special knowledge gleaned during workup and to appraise the patient of it when appropriate.

\section{Operative Procedure}

Thyroidectomy commences with induction of general anesthesia. There is a limited but useful place for local anesthesia during thyroidectomy. In our experience total thyroidectomy for amiodarone-induced thyrotoxicosis (AIT) has been performed using local anesthesia without incident. Ideally, at the time of induction, the surgeon is in the immediate vicinity, as the anesthesiologist may need assistance at the time of endotracheal intubation.

Positioning the patient on the operating table is important to the patient and the operating team. The patient must be positioned to an optimal level of neck extension by placing an appropriate sandbag or pillow underneath the shoulders; this maneuver extends and facilitates surgical access to the neck. On occasion, the degree of kyphosis in elderly patients does not allow extension of the neck. Extension should not be excessive, particularly in elderly patients with degenerative disease of the cervical spine. Apart from local pain in the neck, severe postoperative frontal or occipital headache, which may persist for several weeks before resolving, can follow hyperextension, regardless of age [9]. It is prudent to place the occiput on a soft foam "donut" to prevent unexpected side-to-side movement during surgery and to offer some support to the extended head.

\section{Skin Incision}

Appropriate placement of the skin incision when securing access to the thyroid gland should ensure adequate exposure for safe surgery to be undertaken [3]. In most patients, the skin incision ranges from one and one-half to two fingerbreadths above the clavicle in a curvilinear fashion within or parallel to a natural skin line.

\section{Recurrent Laryngeal Nerve}

Injury to the recurrent laryngeal nerve (RLN) should occur in fewer than $1 \%$ of patients undergoing thyroidectomy. Although prevention of injury can never be absolute, careful observation during every operative step to monitor the wide range of possible variations in the course of the nerve should substantially reduce risk $[10,11]$. Whether the RLN should always be identified during performance of a total or subtotal lobectomy has been the subject of considerable debate in the past. Some skilled, experienced surgeons have obtained a low nerve injury rate without deliberate routine nerve exposure. The current consensus view is that the nerve is less likely to be injured if clearly visualized during at least a portion of the procedure. Whether the nerve is exposed caudal to the inferior thyroid artery early in the operation or after some mobilization of the superior and inferior poles, medial retraction of the thyroid lobe remains a matter of training, experience, and preference of the individual surgeon [4, 11-13].

The authors do not routinely identify the RLN until the gland has been mobilized, with the exception of the tubercle of Zuckerkandl and the ligament of Berry. In most cases, the distal course of the nerve $(2-3 \mathrm{~cm})$, where injury is most likely to occur, cannot be seen until this point in the procedure regardless of its earlier identification caudally. Most often the distal portion of the nerve is covered by the tubercle of Zuckerkandl [14] or a portion of the ligament of Berry (or both). In our opinion, this is where the nerve is most likely to be at risk during lobectomy (severance, traction, or clamping) or partial resection (ligation).

Early identification of the RLN in its more proximal course does not prevent its injury if any complacency occurs during the dissection in the region of the ligament of Berry. After careful mobilization of the tubercle of Zuckerkandl and its retraction medially, the nerve can always be identified in this area. In most cases the nerve traverses just medial to the tubercle and is hidden from view until the tubercle is medially retracted. This is also the region where on the right side, in approximately $1 \%$ of cases, a nonrecurrent laryngeal nerve or direct branch from the vagus can be identified as well [10]. In our experience this occurs in $0.5 \%$ to $1.0 \%$ of cases. This is not a problem on the left side and has been reported in only the rare patient with situs inversus. Because the 
RLN can bifurcate or even trifurcate proximal to its laryngeal entrance, no portion of the ligament of Berry should be cut or clamped until the surgeon is $100 \%$ sure that a nerve branch is not within the tissue involved.

During the last decade, even when we were sure the nerve was lateral or posterior to the ligament of Berry being transected, we identified the nerve in this area for teaching purposes and have not incurred a permanent nerve injury. This technique usually requires no more than a few moments to do in cases in which the distal nerve was not already exposed after mobilizing the tubercle of Zuckerkandl. It is clear that careful exposure does not cause permanent nerve injury. It should be noted that when performing subtotal lobectomy the distal course of the RLN may not always be visualized, regardless of whether its proximal course is exposed early. It should be assumed that the distal nerve may run medial to or in a crevice on the surface of the remaining remnant, although it is rare. Great care should be taken when placing hemostatic sutures so they are not placed deeply enough to possibly encompass the nerve.

As far as nerve injury is concerned, many experienced surgeons consider total lobectomy a safer procedure than subtotal lobectomy because the distal nerve can always be visualized during total lobectomy. Aside from direct physical injury, the surgeon must always be aware of the potential for nerve injury due to heat from imprudent use of electrocautery anywhere in the proximity of the RLN.

\section{External Branches of the Superior Laryngeal Nerve}

The superior laryngeal nerve, although innervating only one muscle, the cricothyroideus, is important in providing a normal voice. The role of this nerve and its muscle is to tense the vocal cords when they are approximated, providing timbre to the voice. This underpins the capacity to project the voice and to scream. Loss of this function is a frustrating complication for all in whom it is injured, especially mothers of young children [4, 12]. It is more than frustration to the professional whose voice is the source of a career, such as a politician, media announcer, or singer. The external branch has been referred to as "the nerve of Amalita Galli Curci" because of the disastrous termination of this worldclass opera singer's career as a result of her injury after a thyroidectomy for benign disease performed during the 1930s. Dissection of the upper pole of the thyroid gland should be anatomic and should lead to avoidance of this problem in most patients. However, downward or caudal retraction of the upper pole to ligate and divide the individual branches of the superior thyroid vessels causes transient paresis of this nerve in rare cases.

It should be noted that approximately $20 \%$ of patients are at risk for injury of the external branch of the superior laryngeal nerve when the superior thyroid vessels are clamped, divided, and ligated en masse, a technique that was commonly used in an era before the anatomy of the external branch with its variations had been well described. By dividing the individual branches of the superior thyroid vessels as they enter the thyroid capsule, those nerves at potential risk because of their proximity to the artery or even its branches are avoided [15]. When this technique is used, only $20 \%$ to $30 \%$ of external nerves are seen directly. Although some surgeons insist on direct visualization of the external branch of the superior laryngeal nerves in all cases, most surgeons do not do so, considering it unnecessary in most cases where the nerve is not at risk at all. In addition to injury to the nerve by clamping is the potential for paresis because of the imprudent use of electrocautery. This should never be used to correct muscle bleeding in the region of the cricothyroideus muscle or the inferior pharyngeal constrictor. Even when using bipolar cautery, care should be taken. Finally, when dissecting the anterior suspensory ligament from the front of the larynx, great care should be taken to avoid direct injury to the cricothyroideus muscle, which may be firmly adherent to it, particularly in cases of lymphocytic thyroiditis.

\section{Cervical Sympathetic Trunk}

The cervical sympathetic trunk is injured on rare occasions when a retroesophageal extension of a goiter is being dissected. Care must be taken over the prespinal surface when mobilizing the carotid sheath. Horner syndrome results from what is usually an avoidable injury (two cases of Horner syndrome among 10,201 thyroidectomies in our Australian series). This area is almost out of bounds unless there is a significant anatomic abnormality or extension of malignancy.

\section{Esophagus}

The esophagus may be at risk when there is significant displacement of the trachea, leaving the anterior esophageal surface exposed to injury. This risk is greater if the surgeon has difficulty locating the RLN in the presence of altered anatomy as can be seen on occasion in large multinodular goiters.

\section{Carotid Artery}

The carotid artery is rarely at risk of injury, although excessive lateral retraction in an older person with an enlarged thyroid gland may reduce flow to a level where ocular or central nervous system damage may occur. Even if the complication is of a minor degree, it is avoidable if assistants are carefully instructed with regard to the use of retraction and tissue handling, and the surgeon is observant during surgery.

\section{Parathyroid Dysfunction}

The cause of hypoparathyroidism after thyroidectomy is not always readily explicable. There is a risk of iatrogenic injury to the parathyroid glands during any operation in which both lobes of the thyroid gland are explored or resected, although permanent hypoparathyroidism may not be due to direct injury alone. Perhaps the best way to avoid parathyroid damage is to clearly know the anatomy of the glands and their blood supply and make every effort to preserve them in situ or to transplant one or more glands when that is not possible.

Hypocalcemia following thyroidectomy is usually temporary. There is a wide variation in the reported incidence ranging from $1.6 \%$ to $50.0 \%$, although most surgical units experienced in total thyroidectomy report a $2 \%$ or less incidence of persistent dysfunction. The parathyroid glands are each supplied by an end-artery, as described by Halsted and Evans 1907 [16], and its disruption is best avoided by dividing branches of the inferior thyroid artery beyond the parathyroid on the thyroid gland capsule [17]. If the end-artery is damaged, transplantation to a carefully prepared "dry" pocket in the sternocleidomastoid muscle or forearm [18] or 
a subcutaneous location [19] is suggested to be the best option to maintain viability and subsequent function of the parathyroid gland. If a parathyroid gland appears congested or ischemic at the end of surgery, a clear incision in the capsule can liberate excess venous blood and allow flow of arterial blood. If this is observed to satisfaction, the gland should remain in situ. If not, it should be transplanted to an appropriate location without hesitation.

A case can be made for transplanting at least one parathyroid gland, usually that suspected of being the most compromised. In patients undergoing total thyroidectomy. Zedenius et al. [20] found that after transplanting one parathyroid into the sternomastoid in each of 100 consecutive patients undergoing total thyroidectomy, none suffered permanent hypoparathyroidism. A similar experience was reported by Lo and Lam: 0 in 98 patients undergoing thyroidectomy [21]. Although transient hypocalcemia occurred in $21.4 \%$ of their patients, none developed permanent hypoparathyroidism. In their overall series of 271 patients, permanent hypocalcemia occurred only in patients who did not undergo a transplant of at least one parathyroid gland (2.9\%). They emphasized the high incidence of inadvertent removal of one or more parathyroid glands, particularly during thyroid cancer operations, and the need for careful examination of resected specimens to identify these glands and to transplant them. They emphasized selective use of transplantation and found it useful in $36 \%$ of their patients.

Apart from surgical injury, unsuspected excision of one or more parathyroids or metabolic factors such as "hungry bones" associated with thyrotoxicosis may cause postoperative hypocalcemia [22]. Postoperatively, it is important to determine whether patients are normocalcemic the next day. If there has been no major injury or accompanying problem, normocalcemia is highly predictive of the patient remaining so. In the asymptomatic patient with a serum calcium level below $8.0 \mathrm{mg} / \mathrm{dl}$ (normal $8.6-10.2 \mathrm{mg} / \mathrm{dl}$ ), oral calcium supplementation with calcium carbonate, 2 to $3 \mathrm{~g}$ daily, is initiated prior to discharge with careful instructions about reporting signs of tingling or muscle cramps. Symptomatic patients - those with a serum calcium level below $7.5 \mathrm{mg} / \mathrm{dl}$ - are given daily calcium carbonate and the rapid-acting vitamin D analog Rocaltrol, 0.25 to $0.5 \mu \mathrm{g}$ (Roche, Basle, Switzerland). Such patients are kept in the hospital an additional day and then discharged if asymptomatic. Their serum calcium is measured again 2 weeks later; if it is in the normal range, they are quickly weaned off their vitamin D and calcium supplement. Most patients can be discharged the day after a total thyroidectomy; with the use of Rocaltrol, only $10 \%$ require an extra day of hospitalization. Although nearly all patients can be weaned off their vitamin D analog, a small percent require drug therapy for up to 3 months, at which time any patient with initial hypocalcemia should be reevaluated to determine if their recovery has been complete. Failure to detect or treat long-term hypoparathyroidism can result in cataracts and other long-term problems that can be avoided with appropriate medication.

It is not clear in individual patients who have had a parathyroid autotransplant and possible damage to the other parathyroid glands whether it is the transplant or possible supernumerary parathyroids that are providing sufficient function for normocalcemia. However, the use of transplantation as insurance against hypoparathyroidism has both intellectual and practical appeal.

\section{Postoperative Bleeding}

Hemorrhage within a thyroidectomy wound usually occurs within 24 hours of operation, with most significant haematomas becoming apparent with 6 to 8 hours. Acute respiratory embarrassment is potentially lethal unless surgically relieved as an emergency procedure. Tracheostomy is infrequently required in those with insidious hemorrhage resulting in laryngeal edema when the diagnosis has been delayed. Fortunately, death is a rare event, as it should be when skilled surgeons are available and all involved staff are educated and aware of the potential consequences of overlooking this complication.

Prevention is always better than cure, and a well planned history can play a distinct role in preventing hemorrhage. A history of excessive bleeding in association with prior wounding or surgery, a family history of bleeding, or medications that may cause bleeding should be elicited and corrective steps taken where possible. It is essential that patients on warfarin, heparin, or aspirin therapy come to operation appropriately protected and monitored. Meticulous hemostasis is mandatory for the surgeon, and there can be no compromising with this degree of care. Until the operative field is dry, the sandbags should not be removed from underneath the shoulders. Once they are removed and the neck flexed, some of the tension on blood vessels is released and may lead to unexpected bleeding. Further close inspection for bleeding should be undertaken. Hence, the neck should not be closed until the surgeon is convinced it is dry.

Occasionally, the surgeon is uncertain about hemostasis. In those circumstances, the anesthetist can perform a Valsalva maneuver, which increases cervical venous pressure and stresses possible bleeding points, causing bleeding, which can be controlled before closure of the neck.

It has been customary in our Australian unit to line the thyroid bed after excision of the gland with a layer of Surgicel (Ethicon, Somerville, NJ, USA). This practice appears to further reduce capillary ooze and serum collection in the wound.

When the wound is closed, it is a sound move to take a surgical sponge and apply manual pressure across the neck in the line of incision until after the patient is extubated. In our experience there is always a cough at the time of extubation, which elevates venous pressure. The manual pressure helps to avoid the rise in venous pressure at the wound site and hence the risk of bleeding it may provoke.

In a series of 10,201 thyroidectomies prospectively followed in the Department of Surgery, Royal North Shore Hospital database (1957-1997), 124 (1.2\%) required reoperation to control hemorrhage and 31 patients $(0.3 \%)$ required tracheostomy. The number has diminished over the last 10 years.

\section{Muscle Closure}

When closing the transversely divided muscles, closure is not completed laterally, leaving a "weep hole" at each margin; furthermore, the platysma not closed. This form of closure leaves more space for blood to disperse should there be any bleeding. On occasion, this technique reduces the need to reopen the neck. The wound is closed with a subcuticular suture of 4-0 Vicryl (Ethicon), and no drainage is used, the wound is dressed with Steri-Strips (3M, St. Paul, MN, USA). For surgeons who do not divide the strap muscles but merely separate them in the midline and retract 
them laterally, reapproximation in the midline in one or two layers is performed leaving a small weep hole at each end. Additionally, some surgeons routinely reapproximate the platysma muscle and its fascia with interrupted buried sutures. The skin may also be closed with interrupted sutures or staples, which can be removed on the first postoperative day, after which Steri-Strips are placed and removed when the patient returns for the first postoperative visit 2 weeks later.

\section{Complications}

Infection is a rare complication of thyroidectomy and may require local drainage and antibiotic therapy without opening the entire wound. In our experience, infection, usually in the form of wound cellulitis, has occurred in $0.1 \%$ of all patients undergoing thyroidectomy during the past 10 years. None has required reopening the wound. Seromas may cause discomfort and require aspiration for patient comfort. This can be done painlessly by inserting a needle above the incision when there is a palpable fluid collection noted at the first postoperative visit. These usual complications of thyroidectomy can cause significant difficulty for patients and a significant sense of discomfort for the surgeon.

\section{Comment}

Overall the principles of surgery, asepsis, hemostasis, careful dissection, restitution of function where possible, and resuscitation should be followed. When surgeons performing thyroidectomy are knowledgeable about the thyroid gland, its function, and its anatomy and are comfortable with its surgery, complications are low in number. Those that occur are largely correctible, thereby reducing the risk of litigation to a minimum among well informed patients.

It is clear that to achieve all this, surgeons should be appropriately trained, follow best practice, and maintain their level of commitment and good communication until the outcome is ensured. In this way the patient is not only satisfied but appropriately relieved of the problem.

\section{Résumé}

La chirurgie de la thyroïde a lieu dans un espace anatomique complexe et dans lequel un nombre important de fonctions physiologiques et sensitives spéciales est contrôlé. La mortalité de la thyroïdectomie est extrêmement rare mais, si le chirurgien réalisant l'intervention n'est pas bien entraîné, sans connaissances approfondies sur la thyroïde et sa fonction, les résultats ne seront pas très bons. Ne pas observer les principes chirurgicaux de base peut engendrer des problèmes médico-légaux. Il vaut mieux observer ces principes pour éviter ces problèmes. C'est le sujet de cette présentation.

\section{Resumen}

La cirugía de la glándula tiroides se practica sobre una región de anatomía compleja y en la cual está ubicado el control de numerosas funciones vitales y de sentidos especiales. La tiroidectomía rara vez resulta en mortalidad, pero a menos que el cirujano que la practica esté bien adiestrado en la técnica operatoria y sea buen conocedor de la glándula y de sus funciones, anatomía y patología, no se lograrán resultados excelentes. La falla en observar los principios cardinales de la cirugía puede dar lugar a dificultades de orden legal. Bien vale la pena observar tales principios y evitar problemas. Tal es el contexto del presente artículo.

\section{References}

1. Ready, A.R., Barnes, A.D.: Complications of thyroidectomy. Br. J. Surg. 81:1555, 1994

2. Al-Suliman, N.N., Ryttou, N.F., Quist, N., Blichert-Toft, M., Graversen, H.P.: Experience in a specialist thyroid surgery unit: a demographic study, surgical complications and outcome. Eur. J. Surg. 163: 13, 1997

3. Bliss, R.D., Gauger, P.G., Delbridge, L.W.: Surgeon's approach to the thyroid gland: surgical anatomy and the importance of technique. World J. Surg. 24:891, 2000

4. Songun, I., Kievit, J., van de Velde, C.J.H.: Complications of thyroid surgery. In Textbook of Endocrine Surgery, Clark, O.H., Duh, Q.Y., editors, Philadelphia, Saunders, 1997, pp. 167-73

5. Lee, T.C., Coffey, R.J., Currer, B.M., Xiu-Ping, M.A., Canary, J.: Propanolol and thyroidectomy in the treatment of thyrotoxicosis. Ann. Surg. 195:766, 1982

6. Gharib, H., James, E.M., Charbonneau, W., Naesseus, J.M., Offord, P.U., Gorman, C.A.: Suppressive therapy with levothyroxine for solitary nodules: a double blind controlled clinical study. N. Engl. J. Med. 317:70, 1987

7. Werga, P., Wallin, G., Skoog, L., Hamberger, B.: Expanding role of fine-needle aspiration cytology in thyroid diagnosis and management. World J. Surg. 24:907, 2000

8. Jarhult, J., Lindestad, P.A., Nordenstrom, J., Perbeck, L.: Routine examination of the vocal cords before and after thyroid and parathyroid surgery. Br. J. Surg. 78:1116, 1991

9. Carden, E., Popp, R.: Ophthalmic blood pressure in thyroidectomised patients. World J. Surg. 3:223, 1979

10. Hunt, P.S., Poole, M., Reeve, T.S.: A reappraisal of the surgical anatomy of the thyroid and parathyroid glands. Br. J. Surg. 55:63, 1968

11. Kadowaki, M.H., Sclard, C., Kaplan, E.L.: Routine exposure of the recurrent laryngeal nerve is important during thyroidectomy. In Debates in Clinical Surgery, Simmons, R.L., editor, Chicago, Year Book, 1990, pp. $190-207$

12. Newsome, H.H.: Complications of thyroid surgery. In Complications in Surgery and Trauma, $2^{\text {nd }}$ ed., Greenfield, L.J., editor, Philadelphia, Lippincott, 1990, pp. 649-672

13. Thompson, N.W., Demers, M.L.: Expoure is not necessary to avoid the recurrent laryngeal nerve during thyroid operations. In Debates in Clinical Surgery, Simmons, R.L., editor, Chicago, Year Book, 1990, pp. 207-223

14. Pelizzo, M.R., Toniato, A., Gemo, G.: Zuckerkandl's tuberculum: an arrow pointing to the recurrent laryngeal nerve (constant anatomical landmark). J. Am. Coll. Surg. 187:334, 1998

15. Moosman, D.A., DeWeese, M.: The external laryngeal nerve as related to thyroidectomy. Surg. Gynecol. Obstet. 127:1011, 1968

16. Halsted, W.S., Evans, H.M.: The parathyroid glandules, their blood supply, and their preservation in operation upon the thyroid gland. Ann. Surg. 46:489, 1907

17. Reeve, T.S., Delbridge, L., Cohen, A., Crummer, P.: Total thyroidectomy: the preferred option for multinodular goitre. Ann. Surg. 206: 782, 1987

18. Olson, J.A., De Benedetti, M.K., Baumann, D.S., Wells, S.A.: Parathyroid autotransplantation during thyroidectomy results of long-term followup. Ann. Surg. 223:472, 1996

19. Fukuuchi, A., Nishi, T., Hori, R., Matsushita, K., Sugimoto, T., Tagwa, H.: Autotransplantation of parathyroid tissues into subcutaneous abdominal adipose tissue in renal hypoparathyroidism. Asian J. Surg. 21:F20:S10, 1998

20. Zedenius, J., Wadstrom, C., Delbridge, L.: Routine transplantation of at least one parathyroid gland during total thyroidectomy may reduce permanent hypoparathyroidism. Aust. N.Z. J. Surg. 69:794, 1999

21. Lo, C., Lam, K.: Postoperative hypocalcemia in patients who did not undergo parathyroid autotransplantation during thyroidectomy: a comparison study. Surgery 124:1081, 1998

22. See, A.C.H., Soo, K.C.: Hypocalcaemia following thyroidectomy for thyrotoxicosis. Br. J. Surg. 84:95, 1997 